\title{
Clinical practice variation and need for pediatric-specific treatment guidelines among rheumatologists caring for children with ANCA-associated vasculitis: an international clinician survey
}

Clara Westwell-Roper ${ }^{1}$, Joanna M. Lubieniecka², Kelly L. Brown', Kimberly A. Morishita', Cherry Mammen',

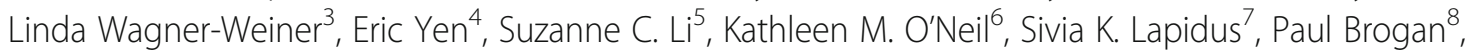

Rolando Cimaz ${ }^{9}$, David A. Cabral ${ }^{*}$ and for ARChiVe Investigators Network within the PedVas initiative

\begin{abstract}
Background: Because pediatric antineutrophil cytoplasmic antibody-associated vasculitis is rare, management generally relies on adult data. We assessed treatment practices, uptake of existing clinical assessment tools, and interest in pediatric treatment protocols among rheumatologists caring for children with granulomatosis with polyangiitis (GPA) and microscopic polyangiitis (MPA).
\end{abstract}

Methods: A needs-assessment survey developed by an international working group of pediatric rheumatologists and two nephrologists was circulated internationally. Data were summarized with descriptive statistics. Pearson's chi-square tests were used in inferential univariate analyses.

Results: The 209 respondents from 36 countries had collectively seen $~ 1600$ children with GPA/MPA; 144 had seen more than two in the preceding 5 years. Standardized and validated clinical assessment tools to score disease severity, activity, and damage were used by 59,63, and 36\%, respectively; barriers to use included lack of knowledge and limited perceived utility. Therapy varied significantly: use of rituximab rather than cyclophosphamide was more common among respondents from the USA (OR $=2.7[1.3-5.5], p=0.0190, n=139)$, those with $>5$ years of independent practice experience $(\mathrm{OR}=3.8[1.3-12.5], p=0.0279, n=137)$, and those who had seen $>10$ children with GPAMPA in their careers (OR $=4.39$ [2.1-9.1], $p=0.0011, n=133$ ). Respondents who had treated $>10$ patients were also more likely to continue maintenance therapy for at least 24 months (OR $=3.0$ [1.4-6.4], $p=0.0161, n=127$ ). Ninety six percent of respondents believed in a need for pediatric-specific treatment guidelines; $46 \%$ supported adaptation of adult guidelines while $69 \%$ favoured guidelines providing a limited range of treatment options to allow comparison of effectiveness through a registry.

Conclusions: These data provide a rationale for developing pediatric-specific consensus treatment guidelines for GPA/MPA. While pediatric rheumatologist uptake of existing clinical tools has been limited, guideline uptake may be enhanced if outcomes of consensus-derived treatment options are evaluated within the framework of an international registry.

Keywords: Pediatric rheumatology, Anti-neutrophil cytoplasmic antibody-associated vasculitis, Granulomatosis with polyangiitis, Microscopic polyangiitis, Physician practice patterns, Clinical practice guidelines, Disease classification, Vasculitis treatment, Outcome assessment

\footnotetext{
* Correspondence: dcabral@cw.bc.ca

${ }^{1}$ Clinical Professor, Division of Rheumatology, Department of Pediatrics,

University of British Columbia, BC Children's Hospital, Room K4-119 4480 Oak

Street Vancouver, Vancouver, BC V6H 3V4, Canada

Full list of author information is available at the end of the article
} 


\section{Background}

Antineutrophil cytoplasmic antibody- (ANCA-) associated vasculitis (AAV) describes the subset of vasculitides primarily involving small vessels: granulomatosis with polyangiitis (GPA, formerly Wegener's granulomatosis), microscopic polyangiitis (MPA), eosinophilic granulomatosis with polyangiitis (EGPA, formerly Churg-Strauss syndrome), and renal-limited pauci-immune glomerulonephritis [1]. Although rare in childhood, AAV carries a high burden of morbidity. With the use of high-dose corticosteroids and cyclophosphamide, AAV is no longer rapidly fatal in the majority of affected children; however, these treatments have significant adverse effects. Balancing the risks associated with existing therapies against the damage associated with under- or overtreatment remains a challenge. In adult populations, this balance has been incrementally fine-tuned through clinical trials that rely on accurate disease sub-classification and scoring tools to stage disease severity, activity, and damage (Table 1). The rarity of pediatric AAV has limited opportunities for pediatric clinical trials such that management decisions are largely informed by adult data [2], with potential for significant practice variation.

A 2005 survey of Childhood Arthritis and Rheumatology Research Alliance (CARRA) members elicited overwhelming consensus on the need to study childhoodonset vasculitis independently from adult disease [3]. ARChiVe (A Registry for Childhood Vasculitis) was established in 2007 based on this imperative for pediatric-specific data collection. A concurrent initiative of the Pediatric Rheumatology European Society (PRES) also helped generate time-of-diagnosis data to support the development of pediatric-specific classification criteria and assessment tools. More recently, PedVas (Pediatric Vasculitis Initiative [4]; https://clinicaltrials.gov/ct2/show/NCT02006134) has allowed collection of clinical data and biological samples to ARChiVe. While pediatric clinical trials remain difficult, an international

Table 1 Definitions of clinical tools included in survey questions

Clinical tool

BVAS Version 3

BVAS for Wegener's Granulomatosis (WG)

Pediatric Vasculitis Activity Score (PVAS)

EUVAS Severity Score

Five Factor Score

Wegener's Granulomatosis Etanercept

Trial (WGET) Severity Score

Disease Extent Index (DEI)

Physician's Global Assessment (PGA)

Vasculitis Damage Index (VDI)

Pediatric VDI (PVDI)

AAV Index of Damage (AVID)
Description

Scoring tool designed to document new or

worseningfeatures of clinically

active AAV. Items are categorized into 9 groups

by organ system [11].

2009 update to BVAS [11, 39].

Modification of BVAS that removes features unlikely

to occur in GPA $[13,40]$.

Pediatric version of BVAS preliminarily validated in children [12].

Classification system for AAV based on disease extent and severity. Subgroups

include localized, early systemic, generalized, severe renal, and refractory disease [41].

Scoring tool based on factors associated with poor prognosis: cardiac symptoms,

gastrointestinal involvement, renal insufficiency, proteinuria, and central nervous

system involvement [42].

Sub-classification based on modified ACR criteria for limited versus extensive disease [43].

Tool for scoring disease activity based on organ system, with distinct domains from those included in BVAS [44].

Physician's global assessment of disease activity on a $10 \mathrm{~cm}$ visual analogue scale.

Scoring tool used to record damage due to disease or treatment. Items are categorized

into 11 groups by organ system with binary scoring [14].

Pediatric modification of the adult VDI; not yet formally validated in children $[14,15]$.

Tool for grading disease- or treatment-associated damage, with weighting of items based

on potential morbidity and mortality [45].

Combination of items from AVID and VDI, divided into 17 organ-based categories [46]. 
registry such as ARChiVe might provide the opportunity to compare effectiveness of a limited range of standardized treatment options and ultimately generate evidencebased guidelines. This strategy has been pursued for uvenile dermatomyositis, another rare pediatric rheumatic disease [5].

As a first step toward developing treatment guidelines for pediatric AAV, we sought to better understand the diversity of beliefs and practices associated with current care. We conducted an international needs assessment survey of physicians involved in the diagnosis and management of children with GPA and MPA, with the following aims: (1) to assess the level of community experience with pediatric AAV; (2) to assess uptake of existing classification criteria, clinical scoring tools, and treatment guidelines; (3) to assess the extent of variation in current treatment practices; and (4) to determine interest in and capacity for use of pediatric treatment protocols.

\section{Methods}

A survey draft developed by the Pediatric Rheumatology group and a nephrologist at British Columbia Children's Hospital was finalized with input from the Vasculitis Working Groups of CARRA and PRES. The survey comprised 47 predominantly categorical multi-choice questions in three sections (Additional file 1). The first section addressed practice type and experience with pediatric GPA and MPA (EGPA was excluded given its rarity in pediatrics). Respondents caring for fewer than two children with GPA and/or MPA over the past 5 years were then given the option to exit. The second section queried use and knowledge of classification criteria and formal scoring tools for disease severity, activity and damage (Table 1). Practitioners were also asked about their use of adult treatment guidelines and perceived need for pediatric-specific guidelines. The third section queried motivations for and barriers to participation in clinical registries. Levels of assigned value were requested using a five-point Likert scale from 1 (not important) to 5 (extremely important).

Members of CARRA, the Canadian Alliance of Pediatric Rheumatology Investigators (CAPRI), the Australian Pediatric Rheumatology Group, the PRESCARRA Vasculitis Working Group, and the Pediatric Rheumatology Bulletin Board (ped-rhe-list@mcmaster.ca) were invited by email to complete the web-based survey (estimated 500 personal email invitations). The survey was administered using REDCap electronic data capture tools [6] hosted at the University of British Columbia (UBC) from July 24 to September 29, 2015. The survey was conducted as a quality assurance initiative for PedVas, which was approved by the Children's and Women's Research Ethics Board of UBC (H12-
00894). Under Article 2.5 of the Tri Council Policy Statement, quality assurance/improvement activities are not subject to further institutional review.

Descriptive statistics were used to quantify response frequencies and means. Unless otherwise specified, frequencies are reported relative to the total number of respondents completing each question. Odds ratios (OR) were determined as measures of association. Limits of 95\% confidence intervals of OR are reported in square brackets. Inferential univariate analysis with two-sided Pearson's chi-square tests and Benjamini-Hochberg correction for multiple comparisons was used to test 11 hypotheses with a false discovery rate of 5\% [7]. Corrected $p$-values are reported. Analysis was performed using Microsoft Excel Version 14.6.7 and Prism Version 5.0a (GraphPad Software Inc., La Jolla, CA).

\section{Results}

\section{Respondent characteristics}

Of 216 respondents opening the survey, 209 completed it, yielding an estimated response rate of approximately $40 \%$. Sixty-five (31\%) chose to exit as they had not cared for two or more patients with GPA/MPA in the preceding 5 years. The 144 respondents completing the full survey practiced in 36 countries, predominantly the USA (48\%), with others from Canada (13\%), Italy (4\%), Australia (3\%), Germany (4\%), Turkey (4\%), United Kingdom (4\%), Brazil (2\%), and Sweden (2\%). Most respondents (92\%) belonged to one or more national or international rheumatology organizations, including CARRA (56\%), PRES (32\%), CAPRI (10\%), the PRES-CARRA Vasculitis Working Group (10\%), and others (18\%). Approximately half of respondents had practiced for less than 10 years after formal training (21\% for less than 5 years and 26\% for 5-10 years); among the rest, $20 \%$ each had practiced for $10-20$ and $20-30$ years and $10 \%$ for $30-$ 40 years. The combined lifetime experience of respondents was at least 1600 patients with GPA/MPA (some shared): $47 \%$ had seen fewer than ten patients, $39 \%$ had seen $10-20$, and only $14 \%$ had seen more than 20 . The majority of respondents $(61 \%)$ had seen five or fewer patients with a new diagnosis of GPA or MPA within the past 5 years (mode 3, median 5, IQR 3-8 patients).

Most respondents belonged to group practices, sharing diagnostic and treatment decisions for all patients (46\%) or managing patients independently while sharing oncall responsibilities (41\%). A minority worked in a solo practice $(7 \%)$ or in another practice arrangement $(6 \%)$, predominantly hospital-based. For patients who had also been assessed by a nephrologist, the majority of respondents reported making collaborative treatment decisions, $30 \%$ in a combined nephrology/rheumatology clinic and $37 \%$ in separate clinic settings. Some reported variation in the responsible subspecialty depending on factors such as who had seen the patient first (18\%), while 13 
and $2 \%$ of respondents reported independent management by rheumatology or nephrology, respectively.

\section{Classification criteria}

There were between 129 and 144 responses (89-100\%) to subsequent individual questions regarding diagnosis and management. While the majority (73\%) reported that they always sub-classify AAV as either GPA or MPA, 26\% endorsed only sometimes sub-classifying. Table 2 shows the number of respondents familiar with - and using - existing criteria for defining or classifying AAV. Most were familiar with ACR1990 [8] and EULAR/PRINTO/PRES 2008 [9] classification criteria for GPA, and with CHCC 1994 disease definitions [10]. Serology (cANCA/PR3 versus pANCA/MPO) and EULAR/PRINTO/PRES 2008 criteria [9] were most frequently used for distinguishing between GPA and MPA in clinical practice. Notably, $66 \%$ of all respondents reported using more than one set of criteria, and those using informal methods considered histopathology, antibody status, and specific organ involvement. The rationale for sub-classification was predominantly prognostication (73\%), influence on treatment choice (48\%), involvement in clinical studies/trials (39\%), and access to treatments (29\%). All respondents felt that distinguishing between GPA and MPA was at least somewhat important for research (mode 4, median 4, IQR 3-4).

\section{Disease severity, activity, and damage}

The proportions of respondents using formal tools to assess disease severity, activity, and damage are shown in Table 3. While most respondents used formal assessment tools for severity (59\%) and activity (63\%), only $36 \%$ had ever formally assessed disease damage. The Birmingham Vasculitis Activity Score (BVAS) [11], the

Table 2 Familiarity with and use of vasculitis classification criteria and disease definitions to differentiate GPA from MPA in children ${ }^{a}$

\begin{tabular}{lll}
\hline Criteria & Familiar (\%) & Using (\%) \\
\hline EULAR/PRINTO/PRES & 78 & 57 \\
2008 criteria [9] & $N^{\mathrm{b}}$ & 57 \\
ANCA (PR3/MPO) & 72 & 29 \\
ACR 1990 criteria [8, 19] & 62 & 32 \\
CHCC 1994 [10] & 47 & \\
CHCC 2012 [1] & 18 & 5 \\
EMA classification & & \\
algorithm (2007) [22] & & \\
Pediatric Modification of EMA & & \\
Classification algorithm (2012) [21] & & 19 \\
Informal criteria & ND & 1 \\
Other formal criteria & $N^{\mathrm{b}}$ &
\end{tabular}

${ }^{\mathrm{a}}$ Data represent percentage of respondents out of $n=144$ (familiar) or $n=143$ (using)

${ }^{\mathrm{b}}$ No data
Pediatric Vasculitis Activity Score (PVAS) [12], and BVAS for Wegener's Granulomatosis [13] - tools designed primarily for assessment of disease activity were frequently used to stage severity, but tools such as the Five Factor Score and the EUVAS and WGET severity scores were used by a minority. PVAS, BVAS, and physician's global assessment on a $10 \mathrm{~cm}$ visual analogue scale (PGA) were the primary tools used by respondents for scoring disease activity, typically at the time of diagnosis: $39 \%$ at only some follow-up visits, $33 \%$ at all visits, $16 \%$ at prescribed times according to research protocols, and $7 \%$ at the time of diagnosis only. Most respondents felt formal assessment of disease activity was somewhat important to clinical management (mode 3 , median 5 , IQR 3-4), and more important to research (mode 5, median 5, IQR 4-5). Most commonly used tools for scoring disease damage include the Vasculitis Damage Index (VDI) [14] or its pediatric adaptation, PVDI [15]. Similar to scoring of disease activity, respondents assessed disease damage primarily at the time of diagnosis: $35 \%$ at only some follow-up visits, $23 \%$ at prescribed times only, and $31 \%$ at every visit. Respondent rationales for not

Table 3 Use of formal disease severity, activity, and damage scoring tools by survey respondents in the assessment of children with GPA or MPA ${ }^{a}$

\begin{tabular}{|c|c|c|}
\hline Parameter & Scoring tool & Respondents (\%) \\
\hline \multirow[t]{9}{*}{ Severity ${ }^{\mathrm{b}}$} & PVAS [12] & 41 \\
\hline & BVAS [11] & 20 \\
\hline & BVAS for WG [13] & 19 \\
\hline & EUVAS Severity Score [41] & 11 \\
\hline & Five Factor Score [42] & 8 \\
\hline & WGET Severity Score [43] & 7 \\
\hline & Disease Extent Index [44] & 1 \\
\hline & Other & 1 \\
\hline & Never formally assess & 41 \\
\hline \multirow[t]{7}{*}{ Activity $^{c}$} & PVAS [12] & 38 \\
\hline & Physician's Global Assessment & 29 \\
\hline & BVAS [11] & 19 \\
\hline & BVAS for WG [13] & 13 \\
\hline & BVAS version 3 [39] & 6 \\
\hline & Other tool & 0 \\
\hline & Never formally assess & 37 \\
\hline \multirow[t]{6}{*}{ Damage $^{c}$} & Pediatric VDI [15] & 31 \\
\hline & VDI [14] & 10 \\
\hline & AAV Index of Damage [45] & 3 \\
\hline & $\begin{array}{l}\text { Combined Damage Assessment } \\
\text { Index [46] }\end{array}$ & 1 \\
\hline & Other & 0 \\
\hline & Never formally assess & 64 \\
\hline
\end{tabular}

${ }^{a}$ Data represent percentage of respondents out of ${ }^{b} n=142,{ }^{c} n=141$ 
using formal assessment tools are shown in Table 4 and included use of histopathology, lack of effect on management, lack of familiarity, and inconvenience.

\section{Treatment guidelines}

A majority of respondents (53\%) reported using a combination of resources to guide treatment decisions, most commonly EULAR/EUVAS recommendations (24\%). A minority used site-specific standardized protocols (7\%); others used pediatric textbook recommendations (4\%), individualized approaches according to personal interpretation (4\%), or advice from colleagues $(6 \%)$. The majority of respondents (96\%) believed in a need for pediatric treatment guidelines for GPA/MPA. Over half $(58 \%)$ were interested in being involved in the process of consensus guideline development - $43 \%$ through an iterative survey - while others felt they did not have the time $(13 \%)$ or relevant expertise (19\%), or were unsure (9\%). There was no association between group membership and the desire to participate in guideline development $(\mathrm{OR}=1.4[0.4,5.1], p=0.7206, n=131)$, although respondents practicing outside the USA and Canada were more likely to want to participate than those within $(\mathrm{OR}=4.2[1.9,9.0], p=0.0011, n=135)$. Most respondents supported consensus guidelines drafted by an expert group (69\%) and believed in the need for a limited range of options to allow for comparative outcome assessment through a clinical registry (63\%). Slightly less than half $(46 \%)$ felt modification of recommendations

Table 4 Reported rationales against use of formal assessment tools for scoring disease severity, activity, and damage in the assessment of children with GPA or MPA ${ }^{a}$

\begin{tabular}{lll}
\hline Parameter & Rationale against use of tool & Respondents (\%) \\
\hline Severity $^{\text {b }}$ & Use of histopathological findings & 53 \\
& No effect on management & 34 \\
& Lack of familiarity with tools & 26 \\
& No value added beyond clinical judgment & 24 \\
& Inconvenience & 16 \\
& Other & 5.3 \\
Activity $^{c}$ & Lack of familiarity with tools & 47 \\
& Inconvenience & 43 \\
& No effect on management & 22 \\
& Lack of applicability of tools for adults & 20 \\
& No value added beyond clinical judgment & 18 \\
Damage & Lack of familiarity with tools & 58 \\
& Inconvenience & 32 \\
& Lack of applicability of tools for adults & 20 \\
& No effect on management & 18 \\
No value added beyond clinical judgment & 12 \\
\hline
\end{tabular}

${ }^{\mathrm{a}}$ Data represent percentage of respondents out of ${ }^{\mathrm{b}} n=38,{ }^{c} n=51,{ }^{d} n=91$ for adult disease was an acceptable method for generating pediatric treatment guidelines.

\section{Treatment practices}

All respondents followed a remission-induction and remission-maintenance model, switching from induction to maintenance therapy within 3-6 months. In adult studies, choice of induction agent may be adjusted based on measures of disease severity, primarily to limit use of aggressive life-saving treatment - specifically cyclophosphamide - that also has significant toxicity and may be unwarranted in milder disease. Two-thirds $(67 \%)$ of respondents endorsed always using more aggressive treatment for patients with severe disease, while $32 \%$ reported only sometimes choosing induction therapy based on disease severity. Table 5 shows first-line induction and maintenance therapies preferred by respondents for treatment of AAV; respondents were not asked to distinguish between GPA- and MPA-specific regimens. In patients with severe disease not requiring intensive care, cyclophosphamide (CYC) was the first choice of remission-induction agent by $66 \%$ of respondents, while $31 \%$ stated they would choose rituximab (RTX). Use of RTX rather than CYC as an induction agent was more frequently reported by respondents from the USA (OR $=2.7[1.3,5.5], p=0.0190, n=139)$, those with greater than 5 years of experience $(\mathrm{OR}=3.8[1.3$, 12.5], $p=0.0279, n=137)$, and those who had seen more than 10 patients with GPA/MPA in their careers $(\mathrm{OR}=4.39$ [2.1, 9.1], $p=0.0011, n=133)$.

Figure 1 shows the heterogeneity among prescribing practices for both CYC and RTX. Most respondents used CYC for induction until clinically inactive disease up to a maximum of 6 months (69\%), while $16 \%$ treated

Table $\mathbf{5}$ Induction and maintenance agents used by respondents ${ }^{a}$

\begin{tabular}{|c|c|c|}
\hline Indication & Agent & $\begin{array}{l}\text { Respondents } \\
\text { (\%) }\end{array}$ \\
\hline \multirow[t]{3}{*}{ Induction agent in severe disease $\mathrm{e}^{\mathrm{b}}$} & Cyclophosphamide & 66 \\
\hline & Rituximab & 31 \\
\hline & Other & 2 \\
\hline \multirow{4}{*}{$\begin{array}{l}\text { Induction agent if not using CYC } \\
\text { or RTX (e.g. for less severe disease) }\end{array}$} & Methotrexate & 40 \\
\hline & Azathioprine & 30 \\
\hline & Mycophenolate & 25 \\
\hline & Other & 4 \\
\hline \multirow[t]{5}{*}{ Maintenance therapy ${ }^{d}$} & Azathioprine & 45 \\
\hline & Methotrexate & 23 \\
\hline & Mycophenolate & 18 \\
\hline & Rituximab & 11 \\
\hline & Other & 3 \\
\hline
\end{tabular}

Data represent percentage of respondents out of ${ }^{b} n=140,{ }^{c} n=138,{ }^{d} n=141$ 

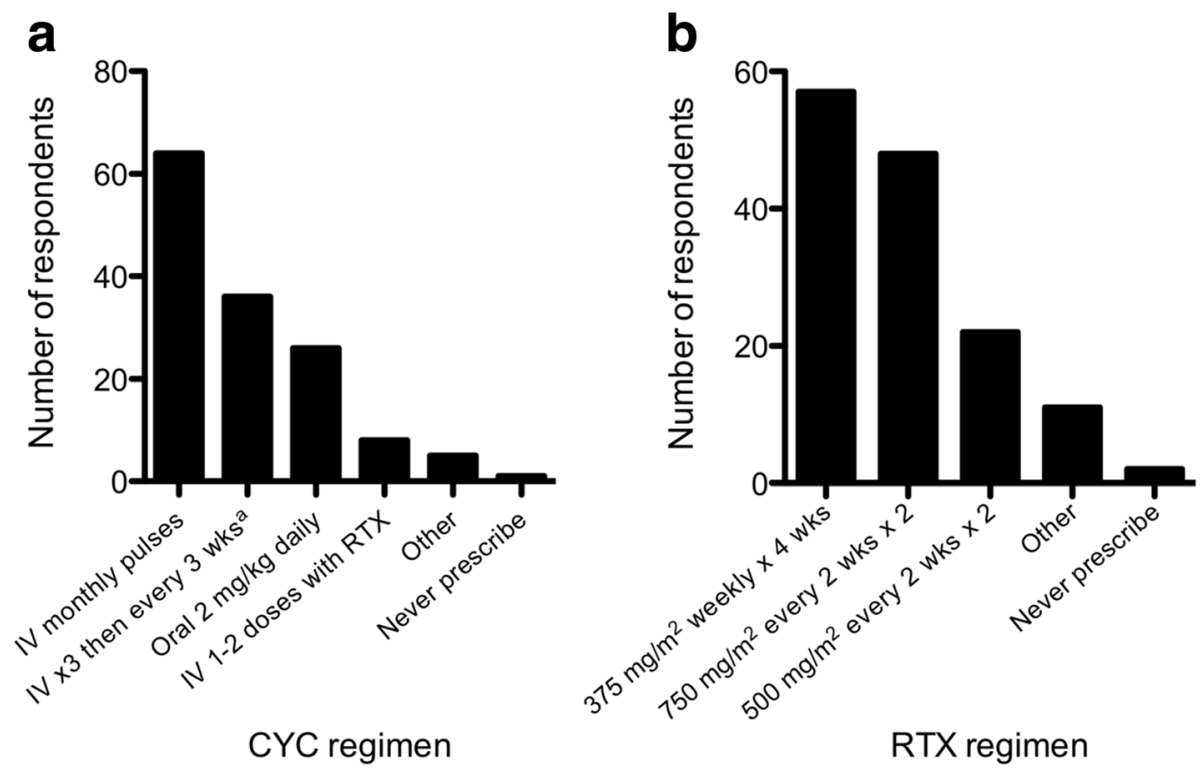

Fig. 1 Typical dosing regimens prescribed for cyclophosphamide (CYC) and rituximab (RTX) induction therapy. Data represent number of respondents out of $n=140$ for both agents. 'EUVAS protocol

for a defined duration between 3 and 7 months, 7\% gave only 1-2 IV doses in conjunction with RTX, 5\% continued induction therapy until clinical inactivity regardless of duration, and $2 \%$ employed some other regimen. The most common first choice of induction therapy in children not receiving CYC or RTX was methotrexate (MTX), followed by azathioprine (AZA) and mycophenolate mofetil (MMF) (Table 5).

The most commonly used remission-maintenance treatment was AZA, followed by MTX, MMF, and RTX (Table 5). There was no association between maintenance therapy and practice location (Canada vs. USA vs. other; $p=0.2009, n=139)$, years of practice $(<5$ vs. 5 or more; $p=0.3042, n=141$ ), or total number of patients seen with GPA/MPA ( $<10$ vs. 10 patients or more; $p=0.8328, n=141)$. The provisional choice for maintenance therapy duration of 24 (46\%), 18 (19\%), 36 $(14 \%)$, or $12(13 \%)$ months was associated with total patient experience: $75 \%$ of respondents who had seen more than 10 GPA/MPA patients in their careers planned for at least 24 months of therapy, compared to $52 \%$ of those who had seen fewer than 10 patients $(\mathrm{OR}=3.0[1.4$, 6.4], $p=0.0161, n=127)$. Duration of maintenance therapy was not associated with practice location (Canada vs. USA vs. other; $p=0.3110, n=130$ ) or years of practice $(<5$ vs. 5 or more; OR $=1.6[0.70,3.89], p=0.3109$, $n=127)$. Concurrent corticosteroid duration initiated at induction was typically six (39\%) or $12(40 \%)$ months, with some respondents continuing for $18(6 \%), 24(4 \%)$, or $36(0.7 \%)$ months and $11 \%$ chose variable periods according to disease severity. $93 \%$ of respondents routinely recommended plasma exchange in certain situations: severe pulmonary hemorrhage and/or rapidly progressive renal disease $(76 \%)$, rapidly progressive renal disease only $(8 \%)$, pulmonary hemorrhage only $(5 \%)$, and with co-existent conditions (10\%).

\section{Interest in clinical registries}

All respondents felt that an international collaborative registry was important for comparative outcome assessment of treatment strategies for GPA/MPA, with $64 \%$ of respondents selecting "very important" (mode 5, median 5, IQR 4-5). Primary motivations for participation in clinical studies or collaborative registries included the potential to improve outcomes for children with AAV (91\%), access to available tools and resources (58\%), endorsement by a formal network of investigators (49\%), potential publication authorship (46\%), and association with specific research objectives (40\%). Only $15 \%$ of respondents felt that a monetary stipend was a major motivation. Registry-associated resources believed to be of most value included an automated PVAS calculator (82\%), an online algorithm to stage disease severity with links to corresponding treatment guidelines (68\%), an automated PVDI calculator (66\%), a classification tool based on entered patient data (54\%), and a printable table to track patient data over multiple visits (54\%). Most clinicians had resources available to assist with the use of registry data entry and clinical tools, including computer and internet access in the clinic (80\% each) and a trainee or fellow (58\%). Fewer had a research assistant (47\%), support for review board applications (37\%), technology support (33\%), or a research nurse (23\%). Major barriers to registry participation included 
insufficient support for data entry (59\%), lack of time (43\%), burden of ethics review board approval (32\%), and lack of patients (24\%).

\section{Discussion}

This survey emphasizes the limited individual experience among clinicians caring for children with GPA and MPA and reiterates clinicians' aspirations to study childhoodonset vasculitis independently from adult disease [3]. International collaborations within the last decade have facilitated the development of pediatric-specific classification criteria for GPA [9] and scoring tools for disease activity and damage $[12,15]$. They have also evaluated the pediatric utility of adult patient algorithms for classifying MPA [16] and staging disease severity [17]. Moreover, the recent revision of the $\mathrm{CHCC}$ definitions is relevant to both adults and children [1]. These newly developed criteria, definitions, and clinical tools are essential for systematic evaluation of drug efficacy. However, the present survey reveals limited uptake by pediatric caregivers and identifies several challenges in the development, implementation, and evaluation of pediatric treatment guidelines.

\section{Distinguishing GPA from MPA}

Most respondents believed that the distinction between GPA and MPA in patients with AAV was important for prognostication, consistent with meta-analyses of adult studies showing lower 5-year survival in MPA versus GPA [18] and of pediatric studies showing less frequent relapses [18] and more severe renal disease [16] in MPA. While respondents were familiar with both the ACR 1990 criteria for GPA [9] and its EULAR/PRINTO/PRES 2008 pediatric adaptation, neither organization includes criteria to define MPA $[8,19]$. Not surprisingly, respondents often used multiple methods to differentiate (subclassify) GPA and MPA phenotypes, and 25\% reported sometimes not differentiating. Frequently, ANCA specificity to PR3 or MPO was used "erroneously" as the primary differentiating criterion to define GPA and MPA, respectively. Adult studies suggest that genetics, pathophysiology, and clinical outcomes may be more strongly associated with antibody status than with the clinical syndromes [20]; although the populations defined by PR3 or MPO antibodies overlap with GPA and MPA, respectively, they are not the same. Indeed, most respondents were not aware of the recently revised CHCC 2012 definitions that do not incorporate ANCA specificity but arguably provide the clearest discriminating definitions of GPA and MPA [1]. Moreover, less than $20 \%$ of respondents were familiar with the European Medicines Agency (EMA) classification algorithm or its pediatric modification [21, 22]. However, this is a complicated tool primarily used in the research setting.

\section{Clinical scoring tools}

Multiple national and international rheumatology organizations have published treatment guidelines for management of AAV in adults [23-31]. Many require formal clinical measurement tools to assess disease "severity" (to stratify appropriate therapy) and "activity" (to determine when to start, stop, or modify therapy). These standardized measures also guide entry of adult patients into research studies and will inevitably guide research eligibility in children. However, one third of respondents reported only sometimes tailoring induction therapy to a formalized disease severity scale, suggesting that other factors - likely including informal assessment, histopathology [32], and specific organ involvement influence management decisions. In children, PVAS [12] may show better correlation with treatment decisions than the BVAS v.3 [33]; nevertheless, equal proportions of respondents used BVAS or its variants. Furthermore, evaluation of permanent damage from disease or treatment is required for outcome assessment and may allow prediction of mortality [34]. The PVDI is a relatively recent pediatric modification of the adult VDI [14] yet to be validated [15]; however, few respondents used either tool. Lack of knowledge or familiarity - as well as perceived lack of utility may account for some lack of uptake. Successful implementation of practice guidelines requiring standardized assessment tools will depend on improved clinician engagement, clear clinical utility, and feasibility of use.

\section{Treatment practices}

Consistent with guidelines for treatment of adults with $\mathrm{AAV}$, all survey respondents employed a remissioninduction and remission-maintenance model. Although $25 \%$ of respondents used EULAR/EUVAS recommendations to guide their treatment decisions, many employed a combination of resources. There was significant variation in medication and dosing regimens for childhood GPA/MPA, with variable use of evidence-based recommendations from existing adult guidelines.

\section{Potential engagement in comparative effectiveness research}

An overwhelming majority of respondents believed in the need for pediatric-specific treatment guidelines and supported guideline development by expert group consensus. It is not simple to reconcile these aspirations with the limited uptake of recent pediatric-specific research and consensus reports, although the described lack of knowledge or familiarity - as well as unclear added benefit beyond current practice - likely contribute. In view of the high frequency of renal disease (75\%) among pediatric AAV patients [16] and the existing high level of cross-specialty collaboration, future efforts towards developing and implementing guidelines for managing patients with AAV must further engage pediatric nephrologists. 
A recent European initiative to develop standards of care for pediatric rheumatic diseases, SHARE (Single Hub and Access point for Paediatric Rheumatology in Europe) [35], will soon provide consensus-based guidelines for management of pediatric vasculitides, including GPA [36]. While many of these recommendations will be based on adult data, most survey respondents favored longer-term comparative outcome assessment of a range of possible consensus approaches. Given barriers to uptake of existing pediatric-specific tools - many of which will be part of standardized guidelines - incentives for implementation are critical. A registry that provides treatment guidelines endorsed by national/international rheumatology organizations, access to clinical tools with automated scoring calculators, and individual patient summary progress reports might further improve engagement and uptake.

Limitations of this study include a sample population that likely underestimates true variability in practice given the selection bias associated with elective survey completion [37] and response rates below 50\% [38]. Participants were reached through formal organizations and therefore may represent a particularly informed group, leading to overestimation of the use of clinical tools and guidelines. Moreover, the survey included few respondents outside Europe and North America, and therefore is limited in generalizability particularly with respect to practice variation in Asian and African countries.

\section{Conclusions}

Taken together, these data suggest a need for and interest in consensus treatment guidelines for pediatric GPA and MPA. They also point to potential challenges associated with guideline development and implementation. Understanding the barriers to uptake of existing classification criteria and formal assessment tools will inform efforts to improve standardization of classification and assessment to guide therapy. Physicians' aspirations for pediatricspecific, evidence-based treatments may motivate uptake of guidelines that evolve from this needs assessment survey and, imminently, from SHARE. Consensus-derived guidelines that include a range of specific treatment options - if provided together with mechanisms for comparative effectiveness research through an international registry - may facilitate clinician engagement and ultimately lead to improved outcomes for children with AAV.

\section{Additional file}

Additional file 1: Copy of Needs-assessment Survey for Treatment of pediatric ANCA-associated vasculitis. (PDF $110 \mathrm{~kb}$ )

\section{Abbreviations}

AAV: Antineutrophil cytoplasmic antibody-associated vasculitis; ANCA: Antineutrophil cytoplasmic antibody; ARChiVe: A registry for childhood vasculitis; AZA: Azathioprine; BVAS: Birmingham vasculitis activity score; CAPRI: Canadian alliance of pediatric rheumatology investigators; CARRA: Childhood arthritis and rheumatology research alliance; CHCC: Chapel hill consensus conference; CYC: Cyclophosphamide; EGPA: Eosinophilic granulomatosis with polyangiitis; EMA: European medicines agency; EULAR: European league against rheumatism; EUVAS: European vasculitis study group; GPA: Granulomatosis with polyangiitis; IQR: Interquartile range; MMF: Mycophenolate mofetil;

MPA: Microscopic polyangiitis; MTX: Methotrexate; PedVas: Pediatric vasculitis initiative; PRES: Pediatric Rheumatology European Society; PRINTO: Paediatric rheumatology international trials organisation; PVAS: Pediatric vasculitis activity score; PVDI: Pediatric vasculitis damage index; RTX: Rituximab; SHARE: Single hub and access point for Paediatric Rheumatology in Europe; VDI: Vasculitis damage index; WGET: Wegener's granulomatosis etanercept trial

\section{Acknowledgements}

The authors thank CARRA, CAPRI, the Vasculitis Working Group of PRES, and the Australian Paediatric Rheumatology Group for input into survey design and/or distribution to their members. CARRA is supported by NIAMS, Friends of CARRA, and the Arthritis Foundation. We also thank Angelyne Sarmiento Rivera and Jayson Tan (British Columbia Children's Hospital) for assistance with REDCap and preliminary data analysis, respectively.

\section{Funding}

This work was supported by a Canadian Institutes of Health Research grant for the PedVas Initiative [TR2-119188 to DAC].

\section{Availability of data and materials}

The survey is attached to this manuscript as a Additional file 1. All data generated or analyzed during this study are included in this published article.

\section{Authors' contributions}

DAC conceived of the study. CW-R, KLB, KAM, CM, and DAC developed the pilot survey. LW-W, EY, SCL, KMO, SKB, PB, RC reviewed, pilot-tested, and modified the survey for the final draft. CW-R analyzed the data with input from JML and prepared the initial manuscript draft. All authors participated in the writing of the manuscript and also read and approved the final draft for submission.

Ethics approval and consent to participate

The survey was conducted as a quality assurance initiative for PedVas, which was approved by the Children's and Women's Research Ethics Board of the University of British Columbia (H12-00894). Under Article 2.5 of the Tri Council Policy Statement, quality assurance/improvement activities are not subject to further institutional review.

Consent for publication

Not applicable

Competing interests

The authors declare that they have no competing interests.

\section{Publisher's Note}

Springer Nature remains neutral with regard to jurisdictional claims in published maps and institutional affiliations.

\section{Author details}

${ }^{1}$ Clinical Professor, Division of Rheumatology, Department of Pediatrics, University of British Columbia, BC Children's Hospital, Room K4-119 4480 Oak Street Vancouver, Vancouver, BC V6H 3V4, Canada. ${ }^{2}$ Simon Fraser University, Burnaby, BC, Canada. ${ }^{3}$ University of Chicago Comer Children's Hospital, Chicago, USA. ${ }^{4}$ University of California - Los Angeles, Los Angeles, CA, USA. ${ }^{5}$ Joseph M. Sanzari Children's Hospital, Hackensack, NJ, USA. ${ }^{6}$ Riley Hospital for Children at IU Health, Indianapolis, IN, USA. ${ }^{7}$ Morristown Medical Center, Morristown, NJ, USA. ${ }^{8}$ Great Ormond Street Hospital NHS Foundation Trust, London, UK. ${ }^{9}$ Ospedale Pediatrico Meyer Firenze, Florence, Italy.

Received: 10 April 2017 Accepted: 1 August 2017

Published online: 07 August 2017

\section{References}

1. Jennette JC, Falk RJ, Bacon PA, Basu N, Cid MC, Ferrario F, Flores-Suarez LF, Gross WL, Guillevin L, Hagen EC, et al. Revised international Chapel 
Hill consensus conference nomenclature of Vasculitides. Arthritis Rheum. 2012;2013(65):1-11.

2. Morishita K, Brown K, Cabral D. Pediatric vasculitis: advances in treatment. Curr Opin Rheumatol. 2015;27:493-9.

3. Wilkinson NM, Page J, Uribe AG, Espinosa V, Cabral DA. Establishment of a pilot pediatric registry for chronic vasculitis is both essential and feasible: a childhood arthritis and rheumatology alliance (CARRA) survey. J Rheumatol. 2007;34:224-6.

4. Cabral D, Benseler S, Foell D, Hancock RE, Ross C, Graham J, Luqmani R. Chronic primary vasculitis in children: a new research opportunity. CRAJ. 2012;22:10-1.

5. Huber AM, Giannini EH, Bowyer SL, Kim S, Lang B, Lindsley CB, Pachman LM, Pilkington C, Reed AM, Rennebohm RM, et al. Protocols for the initial treatment of moderately severe juvenile dermatomyositis: results of a Children's arthritis and rheumatology research alliance consensus conference. Arthritis Care Res. 2010;62:219-25.

6. Harris PA, Taylor R, Thielke R, Payne J, Gonzalez N, Conde JG. Research electronic data capture (REDCap) - a metadata-driven methodology and workflow process for providing translational research informatics support. J Biomed Inform. 2009:42:377-81.

7. Benjamini $Y$, Hochberg $Y$. Controlling the false discovery rate - a practical and powerful approach to multiple testing. J R Stat Soc Series B Stat Methodol. 1995;57:289-300.

8. Leavitt RY, Fauci AS, Bloch DA, Michel BA, Hunder GG, Arend WP, Calabrese LH, Fries JF, Lie JT, Lightfoot RW Jr, et al. The American College of Rheumatology 1990 Criteria for the classification of Wegener's granulomatosis. Arthritis Rheum. 1990;33:1101-7.

9. Ozen S, Pistorio A, lusan SM, Bakkaloglu A, Herlin T, Brik R, Buoncompagni A, Lazar C, Bilge I, Uziel Y, et al. EULAR/PRINTO/PRES criteria for HenochSchonlein purpura, childhood polyarteritis nodosa, childhood Wegener granulomatosis and childhood Takayasu arteritis: Ankara 2008. Part II: final classification criteria. Ann Rheum Dis. 2010;69:798-806.

10. Jennette JC, Falk RJ, Andrassy K, Bacon PA, Churg J, Gross WL, Hagen EC, Hoffman GS, Hunder GG, Kallenberg CG, et al. Nomenclature of systemic vasculitides. Proposal of an international consensus conference. Arthritis Rheum. 1994:37:187-92.

11. Luqmani RA, Bacon PA, Moots RJ, Janssen BA, Pall A, Emery P, Savage C, Adu D. Birmingham Vasculitis activity score (BVAS) in systemic necrotizing vasculitis. QJM. 1994;87:671-8.

12. Dolezalova P, Price-Kuehne FE, Ozen S, Benseler SM, Cabral DA, Anton J, Brunner J, Cimaz R, O'Neil KM, Wallace CA, et al. Disease activity assessment in childhood Vasculitis: development and preliminary validation of the paediatric Vasculitis activity score (PVAS). Ann Rheum Dis. 2013;72:1628-33.

13. Stone JH, Hoffman GS, Merkel PA, Min Yl, Uhlfelder ML, Hellmann DB, Specks U, Allen NB, Davis JC, Spiera RF, et al. A disease-specific activity index for Wegener's granulomatosis: modification of the Birmingham Vasculitis activity score. International network for the study of the systemic Vasculitides (INSSYS). Arthritis Rheum. 2001;44:912-20.

14. Exley AR, Bacon PA, Luqmani RA, Kitas GD, Gordon C, Savage CO, Adu D. Development and initial validation of the Vasculitis damage index for the standardized clinical assessment of damage in the systemic vasculitides. Arthritis Rheum. 1997:40:371-80.

15. Dolezalova P, Wilkinson NW, Brogan PA, Anton J, Benseler SM, Brunner J, Cabral DA, Cimaz R, O'Neil KM, Özen S, Luqmani R. Paediatric Vasculitis damage index: a new tool for standardised disease assessment. Ann Rheum Dis. 2014;73:696-7.

16. Cabral DA, Canter DL, Muscal E, Nanda K, Wahezi DM, Spalding SJ, Twilt M, Benseler SM, Campillo S, Charuvanij S, et al. Comparing presenting clinical features of 48 children with microscopic polyangiitis (MPA) against 183 having granulomatosis with polyangiitis (GPA). An ARChive study. Arthritis Rheumatol. 2016;68:2514-26.

17. Morishita K, Guzman J, Chira P, Muscal E, Zeft A, Klein-Gitelman M, Uribe AG Abramson L, Benseler SM, Eberhard A, et al. Do adult disease severity subclassifications predict use of cyclophosphamide in children with ANCAassociated vasculitis? An analysis of ARChiVe study treatment decisions. J Rheumatol. 2012;39:2012-20

18. Iudici M, Quartier P, Terrier B, Mouthon L, Guillevin L, Puechal X. Childhoodonset granulomatosis with polyangiitis and microscopic polyangiitis: systematic review and meta-analysis. Orphanet J Rare Dis. 2016;11:141.

19. Fries JF, Hunder GG, Bloch DA, Michel BA, Arend WP, Calabrese LH, Fauci AS, Leavitt RY, Lie JT, Lightfoot RW, Jr., et al: The American College of
Rheumatology 1990 criteria for the classification of vasculitis. Summary. Arthritis Rheum 1990, 33:1135-1136.

20. Pepper RJ, Salama AD. Classifying and predicting outcomes in ANCAassociated glomerulonephritis. Nephrol Dial Transplant. 2012;27:2135-7.

21. Uribe AG, Huber AM, Kim S, O'Neil KM, Wahezi DM, Abramson L, Baszis K, Benseler SM, Bowyer SL, Campillo S, et al. Increased sensitivity of the European medicines agency algorithm for classification of childhood granulomatosis with polyangiitis. J Rheumatol. 2012;39:1687-97.

22. Watts R, Lane S, Hanslik T, Hauser T, Hellmich B, Koldingsnes W, Mahr A, Segelmark M, Cohen-Tervaert JW, Scott D. Development and validation of a consensus methodology for the classification of the ANCA-associated vasculitides and polyarteritis nodosa for epidemiological studies. Ann Rheum Dis. 2007;66:222-7.

23. McGeoch L, Twilt M, Famorca L, Bakowsky V, Barra L, Benseler S, Cabral DA, Carette S, Cox GP, Dhindsa N, et al. CanVasc recommendations for the management of antineutrophil cytoplasm antibody (ANCA)-associated vasculitides - executive summary. Can J Kidney Health Dis. 2015;2:43.

24. Lapraik C, Watts R, Bacon P, Carruthers D, Chakravarty K, D'Cruz D, Guillevin L, Harper L, Jayne D, Luqmani $R$, et al. BSR and BHPR guidelines for the management of adults with ANCA associated vasculitis. Rheumatology. 2007:46:1615-6.

25. Ntatsaki E, Carruthers D, Chakravarty K, D'Cruz D, Harper L, Jayne D, Luqmani R, Mills J, Mooney J, Venning M, et al. BSR and BHPR guideline for the management of adults with ANCA-associated vasculitis. Rheumatology. 2014:53:2306-9.

26. French Vasculitis Study Group: Vascularites nécrosantes systémiques Protocole national de diagnostic et de soins. L'Haute autorité de santé de France; 2009 .

27. Charles P, Bienvenu B, Bonnotte B, Gobert P, Godmer P, Hachulla E, Hamidou M, Harle JR, Karras A, Lega JC, et al. Rituximab: recommendations of the French Vasculitis study group (FVSG) for induction and maintenance treatments of adult, antineutrophil cytoplasm antibody-associated necrotizing vasculitides. Presse Med. 2013;42:1317-30.

28. KDIGO Work Group. Kidney Disease: Improving Global Outcomes (KDIGO) Glomerulonephritis Work Group: KDIGO Clinical Practice Guideline for Glomerulonephritis. Kidney Int. 2012;2:139-274.

29. Mukhtyar C, Guillevin L, Cid MC, Dasgupta B, de Groot K, Gross W, Hauser T, Hellmich B, Jayne D, Kallenberg CG, et al. EULAR recommendations for the management of primary small and medium vessel vasculitis. Ann Rheum Dis. 2009;68:310-7.

30. Menahem S, Hiremagalur B, Mudge D, Toussaint N, Walters G. Caring for Australians with renal impairment: the CARI guidelines. Induction and maintenance therapy in ANCA-associated systemic vasculitis. Nephrology. 2008;13(Suppl 2):S24-36.

31. Japanese Circulation Society Joint Working Group: Guideline for management of vasculitis syndrome (JCS 2008). Japanese Circulation Society. Circ J. 2011(75):474-503.

32. Berden $A E$, Ferrario F, Hagen EC, Jayne DR, Jennette JC, Joh K, Neumann I, Noel LH, Pusey CD, Waldherr R, et al. Histopathologic classification of ANCAassociated glomerulonephritis. J Am Soc Nephrol. 2010;21:1628-36.

33. Morishita K, Li SC, Muscal E, Spalding S, Guzman J, Uribe A, Abramson L, Baszis $\mathrm{K}$, Benseler $\mathrm{S}$, Bowyer $\mathrm{S}$, et al. Assessing the performance of the Birmingham Vasculitis activity score at diagnosis for children with antineutrophil cytoplasmic antibody-associated Vasculitis in a registry for childhood Vasculitis (ARChiVe). J Rheumatol. 2012:39:1088-94.

34. Bhamra K, Luqmani R. Damage assessment in ANCA-associated vasculitis. Curr Rheumatol Rep. 2012;14:494-500.

35. Wulffraat NM, Vastert B. SHARE consortium: time to share. Pediatr Rheumatol Online J. 2013;11:5.

36. de Graeff N, Groot N, Kamphuis S, Beresford M. FRI0271 final evidencebased recommendations for diagnosis and treatment of paediatric Vasculitides. Ann Rheum Dis. 2015;74:522-3.

37. Khazaal $Y$, van Singer $M$, Chatton A, Achab S, Zullino D, Rothen $S$, Khan $R$, Billieux J, Thorens $G$. Does self-selection affect samples' representativeness in online surveys? An investigation in online video game research. J Med Internet Res. 2014;16:e164.

38. Edwards PJ, Roberts I, Clarke MJ, DiGuiseppi C, Wentz R, Kwan I, Cooper $R$, Felix LM, Pratap S. Methods to increase response to postal and electronic questionnaires. Cochrane Database of Systematic Reviews. 2009. Issue 3. Art. No.: MR000008. doi:10.1002/14651858.MR000008.pub4.

39. Mukhtyar C, Lee R, Brown D, Carruthers D, Dasgupta B, Dubey S, Flossmann O, Hall C, Hollywood J, Jayne D, et al. Modification and validation of the Birmingham Vasculitis activity score (version 3). Ann Rheum Dis. 2009;68:1827-32. 
40. Mahr AD, Neogi T, Lavalley MP, Davis JC, Hoffman GS, McCune WJ, Specks U, Spiera RF, St Clair EW, Stone JH, et al. Assessment of the item selection and weighting in the Birmingham vasculitis activity score for Wegener's granulomatosis. Arthritis Rheum. 2008;59:884-91.

41. European therapeutic trials in ANCA-associated systemic vasculitis: disease scoring, consensus regimens and proposed clinical trials. European Community study group on clinical trials in systemic Vasculitis ECSYSVASTRIAL. Clin Exp Immunol. 1995;101(Suppl 1):29-34.

42. Guillevin L, Pagnoux C, Seror R, Mahr A, Mouthon L, Le Toumelin P. French Vasculitis study G: the five-factor score revisited: assessment of prognoses of systemic necrotizing vasculitides based on the French Vasculitis study group (FVSG) cohort. Medicine. 2011;90:19-27.

43. Stone JH. Wegener's Granulomatosis Etanercept trial research group: limited versus severe Wegener's granulomatosis: baseline data on patients in the Wegener's granulomatosis etanercept trial. Arthritis Rheum. 2003:48:2299-309.

44. de Groot K, Gross WL, Herlyn K, Reinhold-Keller E. Development and validation of a disease extent index for Wegener's granulomatosis. Clin Nephrol. 2001;55:31-8.

45. Seo P, Luqmani RA, Flossmann O, Hellmich B, Herlyn K, Hoffman GS, Jayne D, Kallenberg CG, Langford CA, Mahr A, et al. The future of damage assessment in vasculitis. J Rheumatol. 2007;34:1357-71.

46. Suppiah R, Flossman O, Mukhtyar C, Alberici F, Baslund B, Brown D, Hasan $\mathrm{N}$, Holle J, Hruskova Z, Jayne D, et al. Measurement of damage in systemic Vasculitis: a comparison of the Vasculitis damage index with the combined damage assessment index. Ann Rheum Dis. 2011;70:80-5.

\section{Submit your next manuscript to BioMed Central and we will help you at every step:}

- We accept pre-submission inquiries

- Our selector tool helps you to find the most relevant journal

- We provide round the clock customer support

- Convenient online submission

- Thorough peer review

- Inclusion in PubMed and all major indexing services

- Maximum visibility for your research

Submit your manuscript at www.biomedcentral.com/submit 